Volume 10, No.2, March - April 2021

International Journal of Advanced Trends in Computer Science and Engineering

Available Online at http://www.warse.org/IJATCSE/static/pdf/file/ijatcse821022021.pdf

https://doi.org/10.30534/ijatcse/2021/821022021

\title{
Experimental Circuit Model for Increasing the Signal Strength level of a Mobile Phone into a Lift
}

\author{
Pankaj Chandra Kar ${ }^{1, *}$, Md. Ariful Islam ${ }^{2}$, Amit Paul ${ }^{3}$ \\ ${ }^{1}$ Department of Information and Communication Technology, Comilla University, Cumilla, Bangladesh \\ ${ }^{2}$ Department of Robotics and Mechatronics Engineering, University of Dhaka, Dhaka, Bangladesh \\ ${ }^{3}$ Department of Physics, Comilla University, Cumilla, Bangladesh \\ pankajkarcouict@gmail.com, arif.rme@du.ac.bd,amitpaulcouphy@gmail.com \\ *Corresponding author
}

\begin{abstract}
Due to rapid change in human lifestyle in recent years, the demand for constructing a building is very high. On the other hand due to the construction of buildings the signal strength level into the lift of that building is very low which is known as the transmission path loss. Path loss may be arising due to reflection, refraction and scattering. Path loss is a function of frequency and time. An innovative circuit model is developed for predicting wireless signal coverage into the lift. In this paper, we propose a simulated circuit model to reduce the penetration loss based on the measurement of window size, temperature, and humidity conditions etc. An outdoor antenna is used to receive the electromagnetic signals from the surrounding environment. The received signals are oscillated by the oscillator circuit. After this, it is amplified and converted into an optical signal. For better performance, this signal is transmitted through the optical fiber. The optical fiber is entered into the wire which is used to move (up-down) the lift. At the last end of the fiber, it is connected to a device which converts this optical signal into an electrical signal. This signal is sent to the indoor model and after amplifying the signal it is transmitted through the indoor antenna. The mobile phone antenna receives that signal and finally it is amplified and sent to the speaker of the mobile phone. This model works on $4 \mathrm{G} / 3 \mathrm{G} / 2 \mathrm{G}$ networks. Our model enhanced the signal strength level in the lift, which is the same outside the lift. It also measured that the signal strength level was the same at the top and ground floor. Required transmitting power is lower than previous methods.
\end{abstract}

Key words: Electromagnetic (EM) wave propagation, Antenna, Resonant Circuit, Amplifier Circuit, Signal Distortion, Power dissipation.

\section{INTRODUCTION}

When an electromagnetic signal passes through any obstacle or it penetrates any building its strength decreases. Increasing rate of the building in our country is very high. Number of obstacles is proportional to the number of constructed building. Electromagnetic waves are emitted by electrically charged particles undergoing acceleration [1], [2] and these waves can subsequently interact with other charged particles, exerting force on them. EM waves carry energy, momentum and angular momentum away from their source particle and can impart those quantities to matter with which they interact. Electromagnetic waves are radiated due to the variety of applications, e.g. communications, radar, remote sensing heating, medical application, etc. Electromagnetic waves are propagating through the space, carrying electromagnetic radiant energy. In the electromagnetic waves the electric and magnetic field vectors are perpendicular to each other in an electromagnetic wave. These waves pass through the antenna of that device and establish a current in the inductor against the back emf or when it is passed through the inductor a steady magnetic field is produced along the axis of the coil [3]. Electric field and magnetic field are perpendicular to each other and their resultant field (electromagnetic field) is perpendicular to both of them. Electric and magnetic fields obey the properties of superposition. Thus, a field due to any particular particle or time-varying electric or magnetic field contributes to the fields present in the same space due to other causes. Further, as they are vector fields, all magnetic and electric field vectors add together according to vector addition [4].

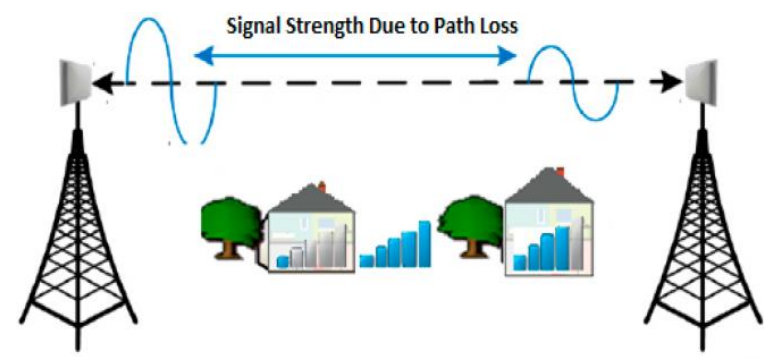

Figure 1: Propagation of Electromagnetic wave [5]

Figure 1 shows that the strength of the electromagnetic signal is decreasing due to path loss.

Despite their significance for coverage forecasts, the estimated model and its simulated circuit model for increasing the signal strength level of a mobile phone into a lift are rare in the literature [6-14]. Penetration loss is reduced by using the previous methods. But still now call drop 
occurs. Most of the time, emergency signals are shown on the mobile phone. No paper gives a clear concept of this proposed model. This paper presents a simulated circuit model for increasing the signal strength level into a lift at $2 \mathrm{G} / 3 \mathrm{G} / 4 \mathrm{G}$ networks. Digital Video Broadcasting Terrestrial/Handheld (DVB-T/H, $600 \mathrm{MHz}$ ), and Global System for Mobile Communications (GSMC) are two frequency bands that are commonly used in today's wireless networks. The outdoor antenna received all the incident electromagnetic signals. A resonant circuit is used to receive only a specific signal which is essential for communication. To keep high signal strength in the lift, optical fiber is used between outdoor and indoor models.

In Section II, a literature review of the proposed model is described. In Section III, key factors and a circuit model of this work are described. In Section IV, simulated results are presented. Section V compares our penetration loss results to previous measurements from the literature. Finally, conclusions are drawn in Section VI.

\section{LITERATURE REVIEW}

The measurements and models could assist the creation of futuristic outdoor-to-indoor residential communication systems for wireless Internet access, wireless cable delivery, and wireless local loops, according to [6] they measured data and empirical models for $5.85-\mathrm{GHz}$ radio propagation path loss for propagation prediction. For typical indoor walls, frequency characteristics of the penetration loss for reinforced concrete walls were calculated over the frequency range of $900 \mathrm{MHz}$ to $18 \mathrm{GHz}$ [7]. At 600, 900, 1800, and $2400 \mathrm{MHz}$, measurements and simulations of vehicle penetration loss (VPL) are presented at illuminated vehicle side, and in-vehicle antenna orientation [8]. millimeter-wave (mmWave) penetration loss measurements and analysis in indoor building materials such as glass doors, glass windows, wardrobe doors, steel doors, and whiteboard writing walls using a wideband sliding channel sounder at $73 \mathrm{GHz}$ [9]. For the design and implementation of future millimeter wave mobile communication networks in New York City, reflections from walls and buildings, as well as penetration losses, were calculated at $28 \mathrm{GHz}$ for indoor and outdoor materials such as tinted glass, transparent glass, stone, concrete, and drywall [10]. A distributed measurement technique and setup that allows for calculating the penetration loss of normal and prototypical railroad car configurations at $800-2600 \mathrm{MHz}$ for azimuthal angles of arrival ranging from 0 to 60 degrees [11]. The investigation of radio transmission into buildings to calibrate such propagation models to develop the indoor field strength coverage based on the outdoor coverage [12]. For the frequency range of 800 to $2000 \mathrm{MHz}$, a penetration model for externally lit buildings in urban areas is proposed [13]. Penetration loss at $28 \mathrm{GHz}$ for fixed wireless Access in a New Jersey suburban residential area [14]. For path loss prediction several low frequency models have been developed. Concerning the related models, class B and class $\mathrm{C}$ amplifier circuits are used [15]. Class B amplifier is a power amplifier where the transistor conducts only half cycle of the input signal. The study of audio power amplifiers is normally done under the assumption that they operate with sine-wave signals. The audio power amplifiers, on the other hand, only work with music and speech signals, such as a musical program, speech, and acoustic noise [16], [17], [18], [19], [20], [21], and [22].

\section{METHODOLOGY}

\section{1 key factors of amplifying EM signal}

\subsubsection{Frequency of the Oscillation

$$
\begin{aligned}
& \omega_{0}=2 \pi f \\
& f=\frac{\omega_{0}}{2 \pi}=\frac{1}{2 \pi \sqrt{L C}}[8]
\end{aligned}
$$

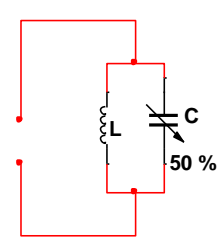

Figure 4: oscillator circuit

Here, $\mathrm{f}=$ frequency of the oscillation

$\mathrm{L}=$ inductance of the oscillation,

$\mathrm{C}=$ Capacitance of the oscillation

\subsubsection{Energy stored in the inductor}

Electromagnetic wave comes from the surrounding environment in establishing the current through the inductor [23], [24].

External power supplied to the inductor,

$\mathrm{P}=|\varepsilon| \mathrm{I}=\mathrm{LI} \frac{\mathrm{dI}}{\mathrm{dt}}$

Energy supplied to the inductor,

$\mathrm{U}_{\mathrm{B}}=\int \mathrm{pdt}=\int_{0}^{\mathrm{I}} \mathrm{LIdI}=\frac{L I^{2}}{2}$

\subsubsection{Class B amplifier Circuit}

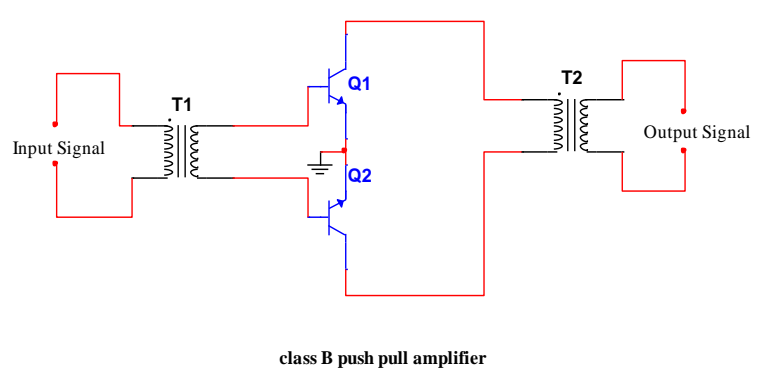

Figure 5: class B push pull amplifier circuit.

In class B amplifier, the Q-point is below the active region. Based on transistor biasing conduction angle is $180^{\circ}$. If we amplify the signal, sometimes the signal may be distorted. To reduce the distortion of the signal class $B$ amplifier is used. Here the output signal from transistor $\mathrm{Q}_{1}$ is $180^{\circ}$ phase of transistor $\mathrm{Q}_{2}$. Due to the cancellation of each other, distortion is reduced. The efficiency of the class B amplifier is low. A 
bypass capacitor is used to stabilize the signal. It eliminates even harmonic in ac output. Due to less distortion, it is used as a radio amplifier. The position of the operating point is on the $\mathrm{x}$-axis.

\subsubsection{Class C amplifier Circuit}

Efficiency of the class $\mathrm{C}$ amplifier is very high. But distortion is very high. As only a small portion of the input signal is amplified therefore the output signal bears very little resemblance to the input signal. Class $\mathrm{C}$ amplifiers have the worst signal reproduction. A resonant circuit is used as a load in class $\mathrm{C}$ amplifier. Conduction angle of class $\mathrm{C}$ amplifier is less than $180^{\circ}$. If the input frequency is same as the resonant frequency then the input signal will amplify. Position of Q-point is below the X-axis or cutoff region. Its efficiency is higher than $80 \%$. It is used for RF amplifier.

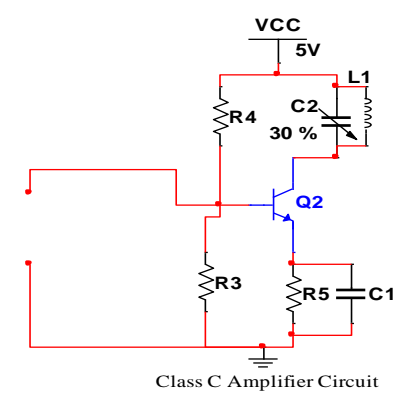

Figure 6: class $\mathrm{C}$ amplifier circuit.

\subsubsection{Amplifier Gain}

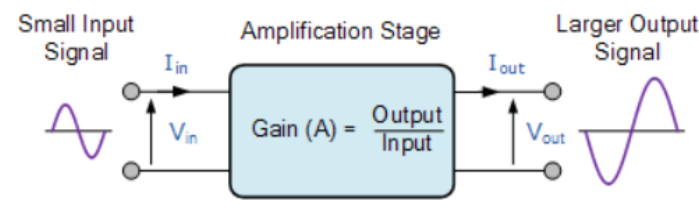

Figure 7: Amplifier gain circuit [25], [26].

Voltage gain, $A_{V}=\frac{\text { output voltage }}{\text { input voltage }}=\frac{V_{\text {out }}}{V_{\text {in }}}$
Current gain, $A_{I}=\frac{\text { output current }}{\text { input current }}=\frac{I_{\text {out }}}{I_{\text {in }}}$

Power gain, $A_{P}=A_{V} \times A_{I}$

\subsubsection{Power Dissipation}

Power dissipation=DC input power $-(\mathrm{DC}$ output power $+\mathrm{AC}$ output power) [27]

\subsection{Signal distortion at class B push pull amplifier}

$y=F(x)$

$\mathrm{i}_{\mathrm{C}}=\sum_{\mathrm{i}=0}^{\mathrm{n}} \mathrm{a}_{\mathrm{i}} \mathrm{x}^{\mathrm{i}}$

$\mathrm{i}_{\mathrm{C}}=\mathrm{a}_{0}+\mathrm{a}_{1} \mathrm{x}+\mathrm{a}_{2} \mathrm{x}^{2}+\mathrm{a}_{3} \mathrm{x}^{3}+\mathrm{a}_{4} \mathrm{x}^{4}+\cdots$
$\mathrm{i}_{\mathrm{C}}=\mathrm{I}_{\mathrm{C}}+\mathrm{a}_{1} \mathrm{i}_{\mathrm{b}}+\mathrm{a}_{2} \mathrm{i}_{\mathrm{b}}{ }^{2}+\mathrm{a}_{3} \mathrm{i}_{\mathrm{b}}{ }^{3}+\mathrm{a}_{4} \mathrm{i}_{\mathrm{b}}{ }^{4}+\cdots$

$i_{C}=I_{C}+a_{1} \cos \omega_{0} t+a_{2} \cos ^{2} \omega_{0} t+a_{3} \cos ^{3} \omega_{0} t+a_{4} \cos ^{4} \omega_{0} t+\cdots$

$\mathrm{i}_{\mathrm{C}}=\mathrm{I}_{\mathrm{C}}+\mathrm{a}_{1} \cos \omega_{0} \mathrm{t}+\frac{\mathrm{a}_{2}}{2}\left(1+\cos 2 \omega_{0} \mathrm{t}\right)+\cdots$

$i_{C}=I_{C}+a_{1} \cos \omega_{0} t+\frac{a_{2}}{2}+\frac{a_{2}}{2} \cos 2 \omega_{0} t+\cdots$

$\mathrm{i}_{\mathrm{C}_{1}}=\mathrm{I}_{\mathrm{c}}+\mathrm{I}_{0}+\mathrm{B}_{1} \cos \omega_{0} \mathrm{t}+\mathrm{B}_{2} \cos 2 \omega_{0} \mathrm{t}+\mathrm{B}_{3} \cos 3 \omega_{0} \mathrm{t}+\mathrm{B}_{4} \cos 4 \omega_{0} \mathrm{t}+\cdots$

$\mathrm{i}_{c_{1}}=\mathrm{I}_{\mathrm{c}}+\mathrm{I}_{0}+\mathrm{B}_{1} \cos \left(\omega_{0} \mathrm{t}+180\right)+\mathrm{B}_{2} \cos 2\left(\omega_{0} \mathrm{t}+180\right)++\mathrm{B}_{3} \cos 3\left(\omega_{0} \mathrm{t}+180\right)+\mathrm{B}_{4} \cos 4\left(\omega_{0} \mathrm{t}+180\right)+\cdots$

$\mathrm{i}_{\mathrm{C}_{2}}=\mathrm{I}_{\mathrm{c}}+\mathrm{I}_{0}-\mathrm{B}_{1} \cos \omega_{0} \mathrm{t}+\mathrm{B}_{2} \cos 2 \omega_{0} \mathrm{t}-\mathrm{B}_{3} \cos 3 \omega_{0} \mathrm{t}+\mathrm{B}_{4} \cos 4 \omega_{0} \mathrm{t}+\cdots$

$\sum \mathrm{i}_{\mathrm{C}}=\mathrm{i}_{\mathrm{C}_{1}}-\mathrm{i}_{\mathrm{C}_{2}}$

$=2 \mathrm{~B}_{1} \cos \omega_{0} \mathrm{t}+2 \mathrm{~B}_{3} \cos 3 \omega_{0} \mathrm{t}+\cdots$

Total output current, $\mathrm{i}_{\mathrm{C}}=\mathrm{I}_{0}+\mathrm{I}_{\mathrm{H}}$

Distortion is

$$
\begin{aligned}
& \mathbf{D}=\sqrt{\frac{\mathrm{B}_{2}{ }^{2}+\mathrm{B}_{3}{ }^{2}+\mathrm{B}_{4}{ }^{2}+\cdots}{\mathrm{B}_{1}{ }^{2}}} \\
& \mathrm{D}=\sqrt{\mathrm{D}_{2}{ }^{2}+\mathrm{D}_{3}{ }^{2}+\mathrm{D}_{4}{ }^{2}+\cdots} \\
& \mathrm{D}_{2}=\left|\frac{\mathrm{B}_{2}}{\mathrm{~B}_{1}}\right|, \mathrm{D}_{3}=\left|\frac{\mathrm{B}_{3}}{\mathrm{~B}_{1}}\right|, \mathrm{D}_{4}=\left|\frac{\mathrm{B}_{4}}{\mathrm{~B}_{1}}\right|
\end{aligned}
$$

Here,

$\mathrm{i}_{\mathrm{C}}=$ Total output current

$\mathbf{i}_{\mathrm{C}_{1}}=$ Current at the collector terminal of the transistor $\mathrm{Q}_{1}$

$\mathrm{i}_{\mathrm{C}_{2}}=$ Current at the collector terminal of the transistor $\mathrm{Q}_{2}$

$\mathrm{D}_{2}=$ Distortion of the $2^{\text {nd }}$ signal

$\mathrm{D}_{3}=$ Distortion of the $3^{\text {rd }}$ signal

$\mathrm{D}_{4}=$ Distortion of the $4^{\text {th }}$ signal

$\mathrm{D}=$ Total distortion of the signal

$\mathrm{I}_{0}=$ Fundamental frequency, $\omega_{0}$

$I_{H}=$ Harmonic component frequency, $\omega_{H}$

In class B push pull amplifier phase difference between the output of transistor $\mathrm{Q}_{1}$ and $\mathrm{Q}_{2}$ is $180^{\circ}$. When the transistor $\mathrm{Q}_{1}$ is on, then the transistor $Q_{2}$ is off or vice versa. Due to the phase difference, the signal is distorted. Even the number of signals is distorted during amplification.

\subsection{Flow chart of the work}

The flow chart by which the proposed model has designed is shown in Figure 3. A tuned circuit is used as a filter to receive the specific signal which is essential for mobile communication. After receiving this tuned signal, it is passed through a class B push pull amplifier circuit to amplify this signal. After amplification, this signal is converted into the optical signal and transmitted through the optical fiber. The last end of the fiber is connected to an optical to electrical signal converter circuit. This converted signal sends to the 
indoor model. The indoor model amplifies it and then it is transmitted through the indoor antenna. The distance between the indoor antenna and the mobile phone antenna is

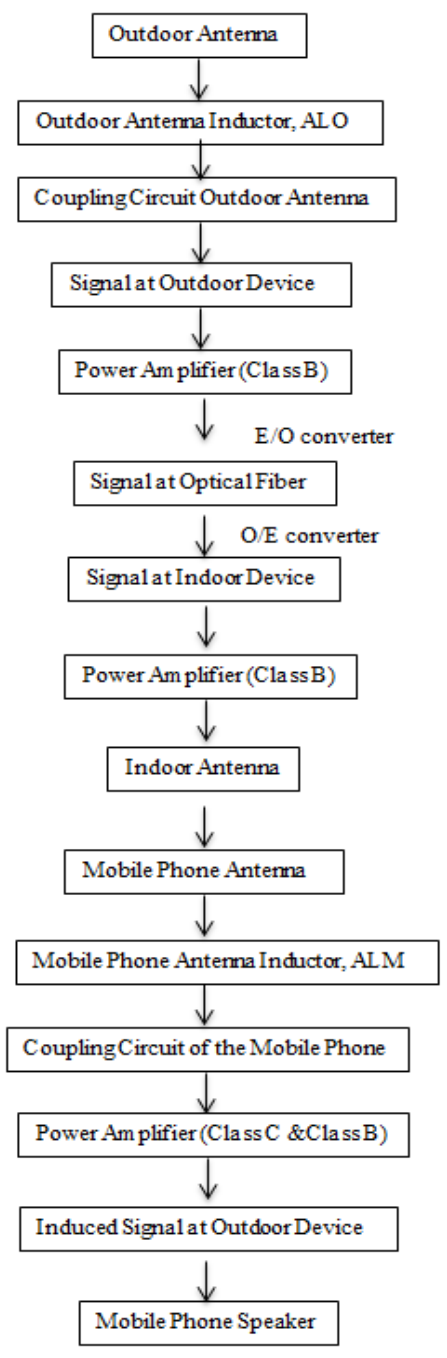

Figure 8: Flow chart of the proposed work

very small, so the required transmitting power level is very low. The mobile antenna receives all the electromagnetic waves and a resonant circuit is used to receive the appropriate signal which indicates that the mobile phone is working on $4 \mathrm{G} / 3 \mathrm{G} / 2 \mathrm{G}$ networks. A class $\mathrm{C}$ amplifier circuit is used to amplify the signal to receive a specific signal of a specific region.

\subsection{Experimental Circuit Design}

The Outdoor antenna receives any kind of electromagnetic signals from the surrounding environment. But all these signals are not suitable for communication. So a resonant circuit is used to receive electromagnetic signals of $4 \mathrm{G} / 3 \mathrm{G} / 2 \mathrm{G}$ networks. Due to path loss power of that signal may be very low. To amplify this signal a class B push pull amplifier circuit is used. Here, ALO is the inductor of the outdoor antenna. TLO and TCO are the tuned inductor and capacitor

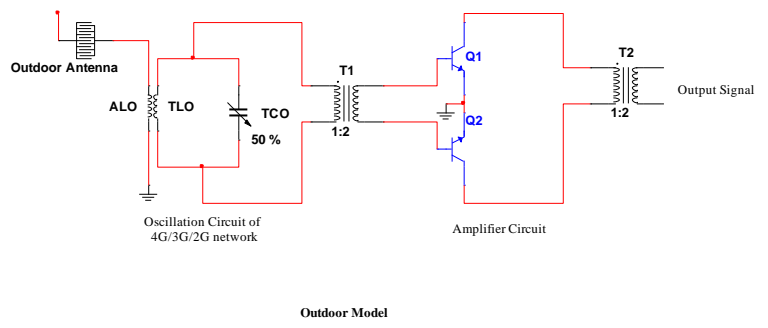

(a)

of the outdoor antenna. By changing the value of the inductor and capacitor, outdoor model can receive specific electromagnetic signals on $4 \mathrm{G} / 3 \mathrm{G} / 2 \mathrm{G}$ networks.

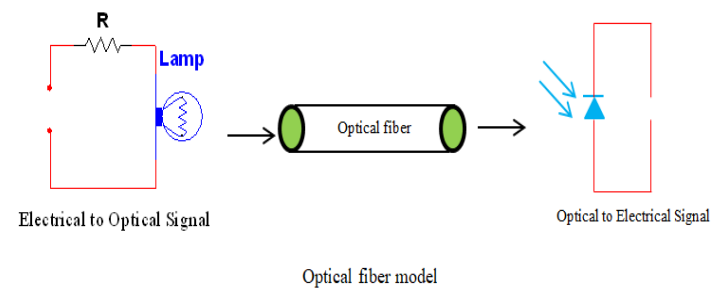

(b)

There are so many obstacles between the outdoor and indoor antenna of this proposed model. To get the perfect level of signal strength into the lift, we used an optical fiber between indoor and outdoor models. Two ends of the fiber are connected to the outdoor and indoor models respectively. To transmit the signal from an outdoor model to an indoor model through the optical fiber, first, the signal is transmitted into an optical signal, passes through the optical fiber and finally converts this optical signal into the electrical signal.

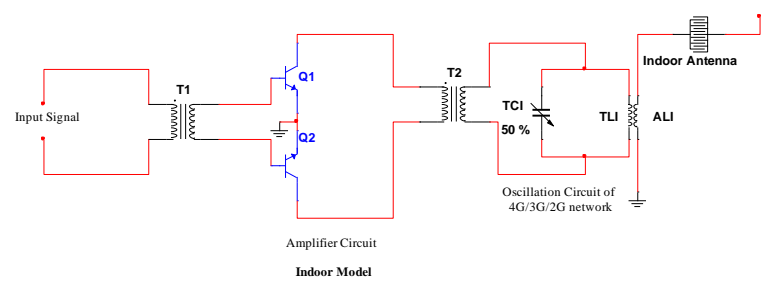

(c)

Normal frequency of any sound is very low and it is totally impossible to send it to a great distance. For transmitting a signal it is modulated. Here the input signal of the indoor model circuit is amplified and using tuned capacitor, TCI and inductor, TLI it is tuned for $4 \mathrm{G} / 3 \mathrm{G} / 2 \mathrm{G}$ networks and finally transmitted to the indoor antenna.

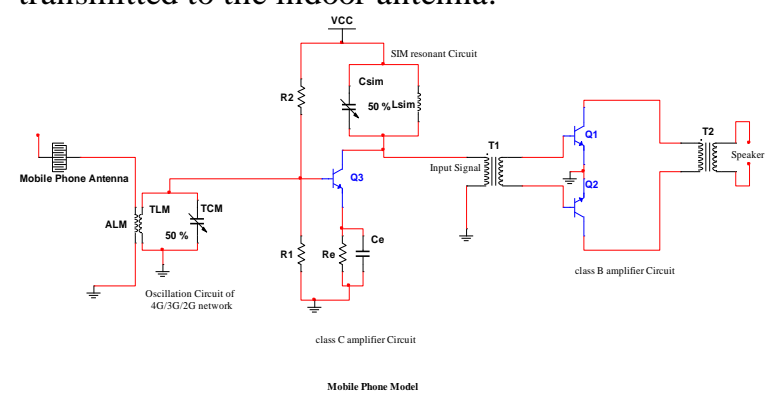

(d)

Figure 9: Schematic receiving circuit diagram (a) outdoor model (b) Optical fiber model (c) Indoor model (d) Mobile Phone model. 
The mobile phone antenna receives any kind of electromagnetic signals from the surrounding environment into the lift. But all signals are not suitable for communication. So a resonant circuit is used to receive electromagnetic signals on $4 \mathrm{G} / 3 \mathrm{G} / 2 \mathrm{G}$ networks. After that for a fixed mobile phone operator a SIM resonant circuit is used. Finally a class B push pull amplifier circuit is used to amplify the signal and after doing it is transferred to the speaker of the mobile phone. Here, ALM is the inductor of the mobile phone antenna. TLM and TCM are the tuned inductor and capacitor of the mobile phone antenna. By changing the value of inductor and capacitor mobile phone can receive a specific electromagnetic signal on $4 \mathrm{G} / 3 \mathrm{G} / 2 \mathrm{G}$ networks. For a specific user the value of Lsim and Csim can be changed.

\section{SIMULATED RESULT}

\subsection{Oscillation of Incident signal at outdoor model}

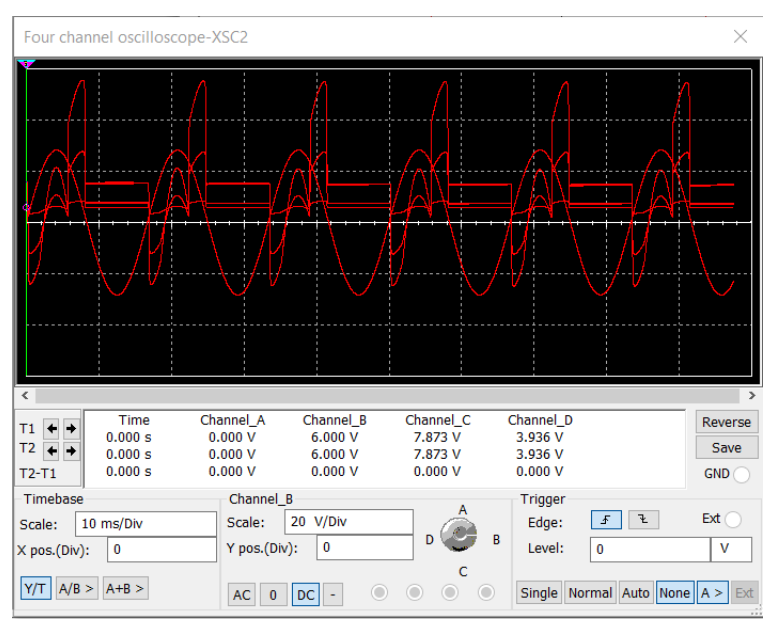

(a)

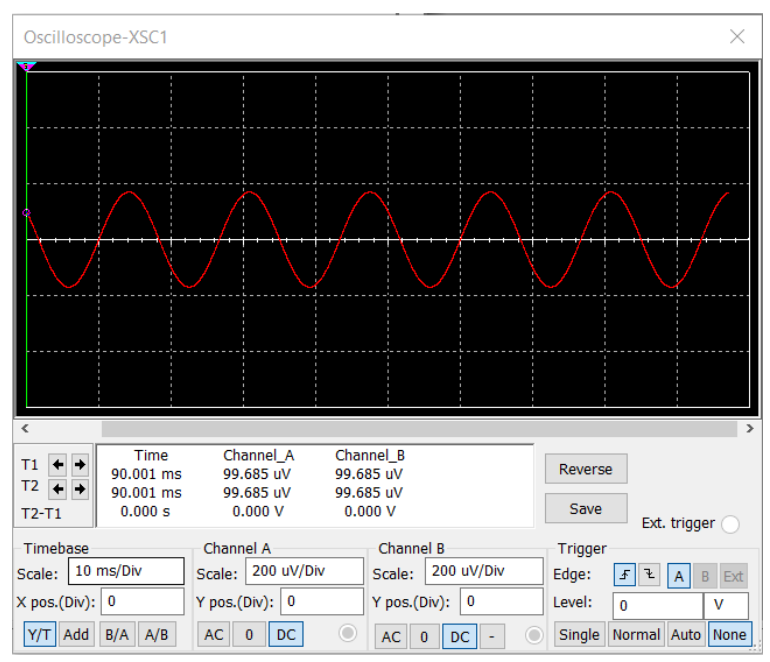

(b)

Figure 10: (a) Incident signal at the outdoor antenna (b) output signal after oscillation at the outdoor model
The outdoor antenna receives all kinds of electromagnetic signals from the surrounding environment. But all of these signals are not suitable for communication. So a resonant circuit is used to pass only a specific signal. In figure 10 (a) depicts a few incident signals and (b) depicts only one signal which is passing through the outdoor model circuit. Other signals do not pass through the circuit. Here, a few signals depict the radiated EM signals from the various electronic devices.

\subsection{Amplification of oscillated signal at outdoor model}

The power of the output signal which passes through the resonant circuit is very low. Before transmitting, it needs to be amplified. Due to the low distortion performance, a class B push pull amplifier is used to amplify the signal. The small signal is the input signal (before amplification) and the big signal is the output signal (after amplification) of the outdoor model.

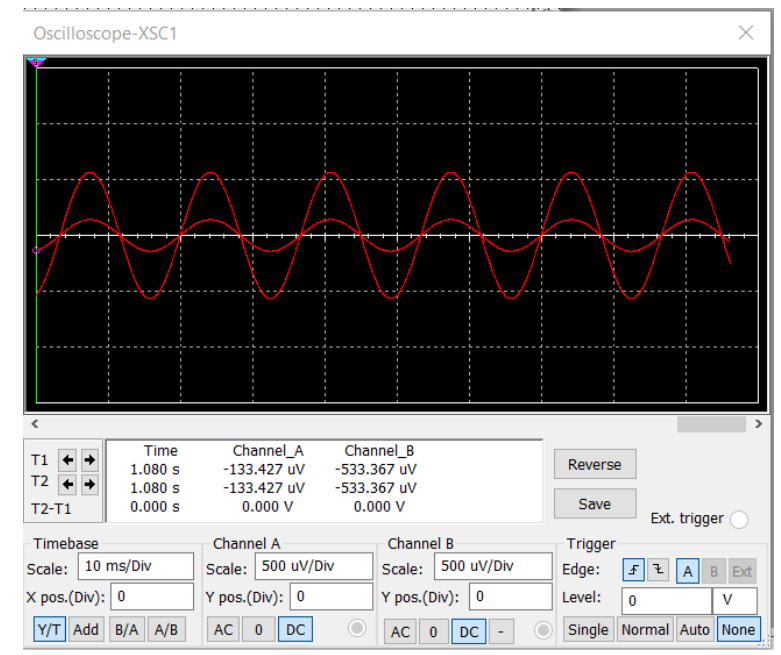

Figure 11: Amplification of oscillating signal using class B push pull amplifier

\subsection{Receiving signal at indoor model}

Before transferring the amplified signal to the indoor model it is converted into the optical signal. During conversion its shape changes due to the dispersion loss. Though the lift moves up and down randomly, number of turns of the fiber varies. If the fiber bends down the reflection occurs at cladding of the fiber. Due to this reason signal shape changes. Signal may be attenuated due to micro bending or macro bending of the fiber. Signal also attenuates due to the dispersion loss. The below figure depicts the signal where dispersion loss is very low. The lower signal is the output signal of the outdoor model and the upper signal is the input signal at the indoor model. 


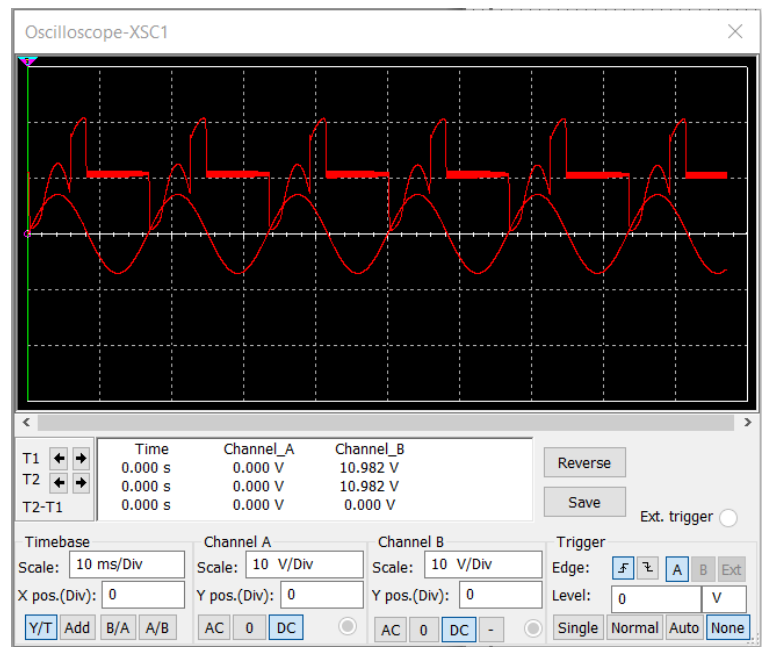

(a) High amount of dispersion loss signal

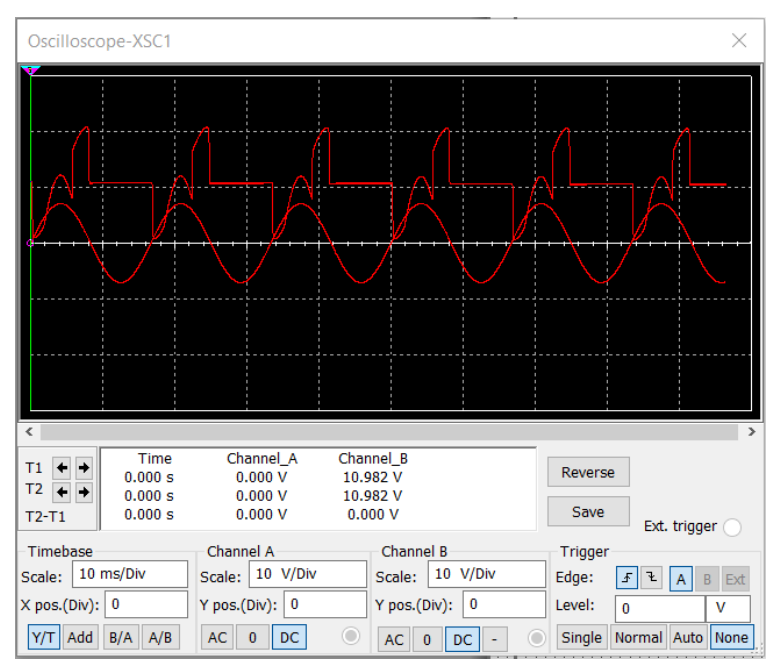

(b) Small amount of dispersion loss signal

Figure 12: Input signal at the indoor model after passing through the optical fiber (a) high amount of dispersion loss (b) small amount of dispersion loss.

\subsection{Amplification of signal at Indoor model}

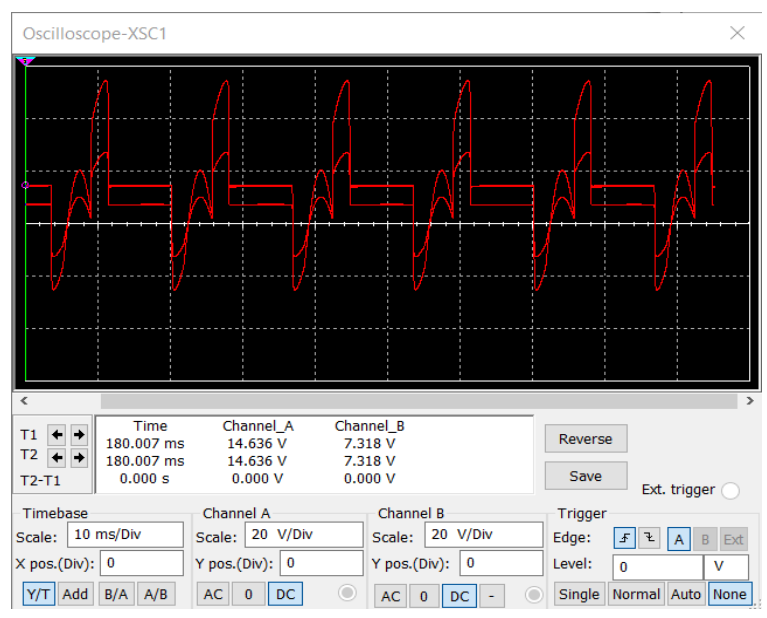

Figure 13: Amplification of signal at indoor model
The indoor antenna circuit will amplify the signal which is fed into the indoor antenna device. After amplifying the signal, it will transmit all around the lift. The transmitting power is very low because the distance between the indoor antenna and the mobile phone antenna is very small. Sometimes signal amplification is not mandatory for great performance. The lower signal is the signal before amplification and the upper signal is the signal after amplification.

\subsection{Oscillation of Incident signal at Mobile Phone model}

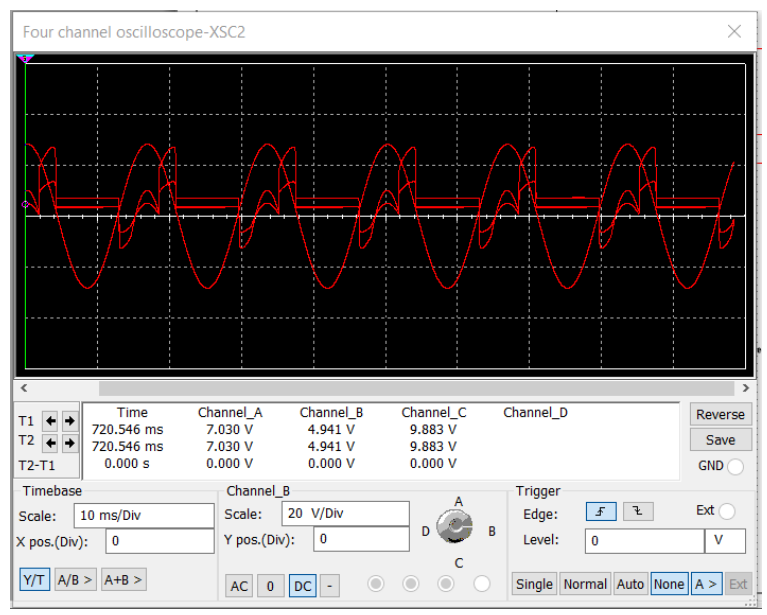

(a)

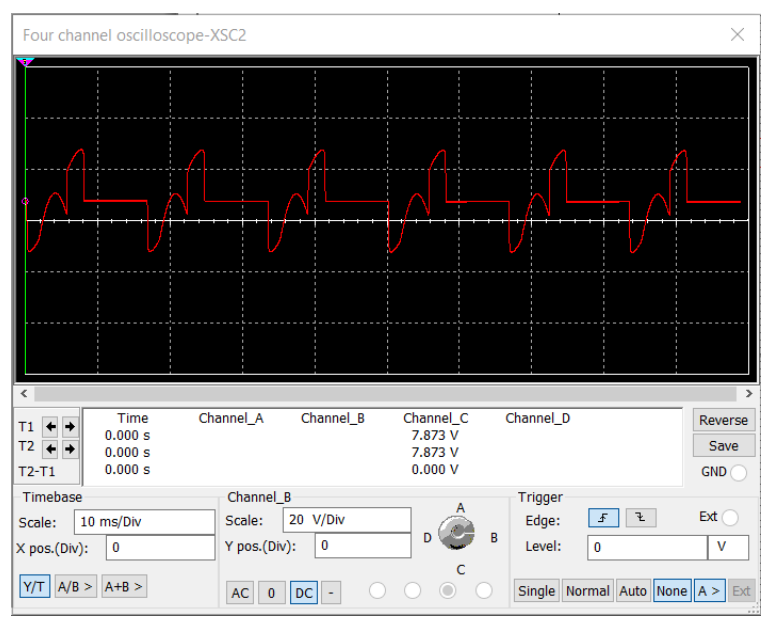

(b)

Figure 14: Incident signal at the mobile phone antenna (b) output signal after oscillation at indoor model.

The mobile phone antennas receive all kinds of electromagnetic signals from the surrounding environment into the lift. But all of these signals are not suitable for communication. So a resonant circuit is used to pass only a specific signal. In figure 14 (a) depicts a few incident signals and (b) depicts only one signal which is passing through the mobile phone model. Other signals do not pass through the circuit. Here a few signals depict the radiated EM signals from the various electronic devices like mobile phones of other people in the lift. The resonating circuit of the mobile 
phone receives only a signal which is perfect for communication on a $4 \mathrm{G} / 3 \mathrm{G} / 2 \mathrm{G}$ networks. Other signals are rejected by the resonant circuit, which is the sim configuration of the mobile phone model.

\section{DISCUSSION}

This work presents a theoretical analysis of the power parameters of class B and class C audio power amplifiers, operating with random sinusoidal signals. In section 3.1.1, parameters of the oscillating circuit are presented. In section 3.2, the parameters of the signal distortion are calculated. Computer simulation with Matlab® and NI Multisim verified the proposed signal strength at different stages. The results obtained can be discussed in several ways: 1) oscillation of incident signal at outdoor antenna 2) amplification of oscillated signal at outdoor model 3) receiving signal at outdoor model 4) amplification of signals on indoor models5) oscillation of incident signal on mobile phone model. This paper is a continuation and a final stage of the exploration of the signal strength amplification into a lift at $2 \mathrm{G} / 3 \mathrm{G} / 4 \mathrm{G}$ networks. Despite high penetration loss, required transmitting power is less than previous methods for a great performance in the lift. In the previous works, the transmitting power was very high. Due to the small distance, the required transmitting power of the indoor antenna signal is very low. So in our prosed method, the possibility of caner is very low. Due to LOS connection the signal strength of the mobile phone into the lift is same as in the outside of the lift. Emergency signal is no founded at anywhere into the lift. Clear concept of dispersion loss is not given in the previous works. In this work, we discussed all the problems related to our work.

\section{CONCLUSION}

The height of the outdoor antenna was greater than the trees surrounding the building. There was a line of sight (LOS) connection between the outdoor antenna and the terrestrial towers. Due to low absorption loss, signal strength remains the same. To receive a specific signal a resonant circuit was used. Induced voltage of the received signal was very low at the inductor of the indoor antenna. Signal distortion was very low in the indoor and outdoor models because class B amplifier was used. Though the signal was passed through the optical fiber there was no scope of occurring electromagnetic interference. So crosstalk was not occurred. Due to the high speed rate of transferring data, the dispersion loss of the signal was very low. The distance between the indoor antenna and the outdoor antenna was very small, so required transmitting power level was very low and it was not mandatory to amplify the signal. The mobile phone antenna also used another resonant circuit to receive a specific signal. The signal strength level in the lift was the same as the outside of the lift. In this proposed method the possibility of cancer was lower than previous.

The major disadvantage is the cross-over distortion.
- Cost of coupling transformer is very high.

- It is difficult to find ideal transformers.

- Transformer coupling causes hum in the output and also affects the low frequency response.

- Transformer coupling is not practical in case of huge loads.

- Due to dispersion loss signal shape must be changed.

\section{FUNDING ACKNOWLEDGEMENT}

This research received no specific grant from any funding agency in the public, commercial, or not-for-profit sectors.

\section{AUTHOR's CONTRIBUTIONS}

P.C.K. conceived the project idea and designed experimental works, conceptualization, edited the manuscript. M.A.I. led the overall research works. M.A.I. supported writing the edited manuscript. A.P. supported writing the preliminary manuscript.

\section{REFERENCES}

1. Cloude, S. (1995). An introduction to electromagnetic wave propagation and antennas. Springer Science \& Business Media.

2. Bettini, A. (2016). A Course in Classical Physics 1--Mechanics. Switzerland: Springer.

3. Purcell, E. M., Purcell, E. M., Purcell, E. M., \& Purcell, E. M. (1965). Electricity and magnetism (Vol. 2, pp. 92-94). New York: McGraw-Hill.

4. Browne, M. (2013). Schaum's Outline of Physics for Engineering and Science. McGraw-Hill Education.

5. Nossire, Z.; Gupta, N.; Almazaydeh, L.; Xiong, X. New Empirical Path Loss Model for $28 \mathrm{GHz}$ and $38 \mathrm{GHz}$ Millimeter Wave in Indoor Urban under Various Conditions. Appl. Sci. 2018, 8, 2122. https://doi.org/10.3390/app8112122

6. G. Durgin, T. S. Rappaport and Hao Xu, "Measurements and models for radio path loss and penetration loss in and around homes and trees at $5.85 \mathrm{GHz}, "$ in IEEE Transactions on Communications, vol. 46, no. 11, pp. 1484-1496, Nov. 1998, doi: 10.1109/26.729393.

7. Y. P. Zhang and Y. Hwang, "Measurements of the characteristics of indoor penetration loss," Proceedings of IEEE Vehicular Technology Conference (VTC), Stockholm, Sweden, 1994, pp. 1741-1744 vol.3, doi: 10.1109/VETEC.1994.345395.

8. E. Tanghe, W. Joseph, L. Verloock and L. Martens, "Evaluation of Vehicle Penetration Loss at Wireless Communication Frequencies," in IEEE Transactions on Vehicular Technology, vol. 57, no. 4, pp. 2036-2041, July 2008, doi: 10.1109/TVT.2007.912164.

9. J. Ryan, G. R. MacCartney and T. S. Rappaport, "Indoor office wideband penetration loss measurements at 73 $\mathrm{GHz}, " 2017$ IEEE International Conference on Communications Workshops (ICC Workshops), Paris, 
France, 2017, pp. 228-233, doi: 10.1109/ICCW.2017.7962662.

10. H. Zhao et al., "28 GHz millimeter wave cellular communication measurements for reflection and penetration loss in and around buildings in New York city," 2013 IEEE International Conference on Communications (ICC), Budapest, Hungary, 2013, pp. 5163-5167, doi: 10.1109/ICC.2013.6655403.

11. M. Lerch, P. Svoboda, S. Ojak, M. Rupp and C. Mecklenbraeuker, "Distributed Measurements of the Penetration Loss of Railroad Cars," 2017 IEEE 86th Vehicular Technology Conference (VTC-Fall), Toronto, ON, Canada, 2017, pp. 1-5, doi: 10.1109/VTCFall.2017.8287899.

12. R. Hoppe, G. Wolfle and F. M. Landstorfer, "Measurement of building penetration loss and propagation models for radio transmission into buildings," Gateway to 21st Century Communications Village. VTC 1999-Fall. IEEE VTS 50th Vehicular Technology Conference (Cat. No.99CH36324), Amsterdam, Netherlands, 1999, pp. 2298-2302 vol.4, doi: 10.1109/VETECF.1999.797348.

13. J. -. Berg, "Building penetration loss along urban street microcells," Proceedings of PIMRC '96 - 7th International Symposium on Personal, Indoor, and Mobile Communications, Taipei, Taiwan, 1996, pp. 795-797 vol.3, doi: 10.1109/PIMRC.1996.568390.

14. J. Du, D. Chizhik, R. Feick, G. Castro, M. Rodríguez and R. A. Valenzuela, "Suburban Residential Building Penetration Loss at $28 \mathrm{GHz}$ for Fixed Wireless Access," in IEEE Wireless Communications Letters, vol. 7, no. 6, pp. 890-893, Dec. 2018, doi: 10.1109/LWC.2018.2835473.

15. Electronics Fundamentals: Circuits, Devices \& Applications. by Thomas Floyd and David Buchla | Jun 23, 2009.

16. Sirakov, E. (2002). The Instantaneous Dissipation of a Class AB Amplifier. RADIOELEKTRONIKA 2002, May 14 - May 16 2002, Bratislava, Slovak Republic. Proceedings of Papers, pp. 457-460.

17. Sirakov, E. (2001). Models of push-pull transistor power amplifier. RADIOELEKTRONIKA 2001, May 10 - May 11 2001, Brno, Czech Republic. Proceedings of Papers, pp. 330-333.

18. Leach, M., Jr. (2010). Introduction to Electroacoustics and Audio Amplifier Design. Dubuque, Iowa Kendall/Hunt Publishing.

19. Amos, S., \& James, M. (2000). Principles of Transistor Circuits. Oxford, Newness.

20. Kazimierczuk, M. (2015). RF Power Amplifiers. Chichester, West Sussex, John Wiley and Sons.

21. Self, D. (2009). Audio Power Amplifier Design Handbook. Oxford, Focal Press.

22. Bortoni, R., Filho, S., \& Seara, R. (2002). On the Design and Efficiency of Class A, B, AB, G, and H Audio Power Amplifier Output Stages. JAES Vol. 50, No. 7/8, pp. 547-563.
23. G. Durgin, T. S. Rappaport and Hao Xu, "Measurements and models for radio path loss and penetration loss in and around homes and trees at $5.85 \mathrm{GHz}$," in IEEE Transactions on Communications, vol. 46, no. 11, pp. 1484-1496, Nov. 1998, doi: 10.1109/26.729393.

24. https://www.electronics-tutorials.ws/amplifier/amplifier -classes.html

25. Electronic Devices and Circuits - RL Boylestad \& Louis Nashelsky.pdf. Electronic Devices and Circuits - RL Boylestad \& Louis Nashelsky.

26. Electronic Principles (SIE) | 7th Edition. by Albert Malvino and David J.

27. Khajeh-Khalili F, Dohni-Zadeh Y (2020) High-Gain Multi-Layer Antenna Using Metasurface for Application in Terahertz Communication Systems. Int $\mathbf{J}$ Electron Device Phys 4:007

28. Bettini, A. (2016). A Course in Classical Physics 1--Mechanics. Switzerland: Springer.

29. C. K. Tse, M. Huang, X. Zhang, D. Liu and X. L. Li, "Circuits and Systems Issues in Power Electronics Penetrated Power Grid," in IEEE Open Journal of Circuits and Systems, vol. 1, pp. 140-156, 2020, doi: 10.1109/OJCAS.2020.3020633S. 